\title{
A pesquisa qualitativa nos programas de pós-graduação em nutrição no Brasil: análise das dissertações e teses
}

\section{Qualitative research in postgraduate programs in nutrition in Brazil: analysis of dissertations and theses}

Francisco de Assis Guedes de

Vasconcelos'

${ }^{1}$ Departamento de Nutrição. Centro de Ciências da Saúde. Universidade Federal de Santa Catarina. Florianópolis, SC, Brasil.

Correspondência / Correspondence

Francisco de Assis Guedes de Vasconcelos

E-mail: fvasconcelos@ufsc.br

\section{Resumo}

O objetivo deste artigo é identificar e analisar as correntes filosóficas e metodológicas do conhecimento científico que embasaram as dissertações e teses com abordagens qualitativas e mistas produzidas nos programas de pós-graduação em Nutrição no Brasil no período de 2003 a 2012. Do universo de 22 programas de pós-graduação vinculados à área de Nutrição, da Coordenação de Aperfeiçoamento de Pessoal de Nível Superior, foram investigados sete programas que dispunham de páginas eletrônicas que disponibilizavam a relação das dissertações e teses. Quanto às abordagens do conhecimento científico, as dissertações e teses foram categorizadas em: abordagens quantitativas, abordagens qualitativas e abordagens mistas. Na análise procedeu-se, além da leitura dos resumos e capítulos metodológicos, a identificação do referencial bibliográfico utilizado, procurando-se identificar: a) O tipo de abordagem ou desenho; b) Os procedimentos e instrumentos do processo de coleta de dados; c) Os procedimentos de análise dos dados; d) Os referenciais teóricos principais e secundários. A hegemonia da abordagem quantitativa, adotada por 92,5\% ( $\mathrm{n}=890)$ do total de dissertações e teses analisadas, denota a forte influência que as correntes filosóficas baseadas no positivismo e nas ciências biológicas continuam exercendo na conformação do campo da nutrição. A participação "marginal" e "emergente" das abordagens qualitativa e mista, adotada por apenas 7,3\% $(n=70)$ do total de dissertações e teses analisadas, expressa a influência que as ciências sociais e humanas e as correntes filosóficas, como a dialética e a fenomenologia, têm exercido sobre um grupo muito restrito de pesquisadores que conformam o campo científico da nutrição.

Palavras-chave: Pesquisa qualitativa. Pós-graduação. Curso de mestrado. Curso de doutorado. Nutrição. Ciências sociais. 


\section{Abstract}

This article intends to identify and analyze the philosophical and methodological trends of the scientific knowledge that were responsible for the dissertations and theses with qualitative and mixed approaches produced by postgraduate programs in Nutrition in Brazil in the period of 2003 to 2012. Of the 22 postgraduate programs that were connected to the area of Nutrition of Coordination for the Development of Higher Education Personnel, seven programs that provide websites with the list of dissertations and theses were investigated. Regarding the scientific knowledge approaches, the dissertations and theses were organized into: quantitative approaches, qualitative approaches and mixed approaches. The analysis proceeded, in addition to reading the abstracts and methodological summaries, to the identification of the bibliographic reference used, seeking to identify: a) The type of approach or design; b) The procedures and instruments of the process of data collection; c) The data analysis procedures; and d) The main and supporting theoretical references. The hegemony of the quantitative approach, adopted by $92.5 \%(n=890)$ of the total dissertations and theses analyzed, shows the strong influence that the philosophical trends based on positivism and biological sciences still have on the field of Nutrition. The "marginal" and "forthcoming" participation of the qualitative and mixed approaches, adopted by only $7.3 \%$ $(n=70)$ of the total dissertations and theses analyzed, expresses the influence that social and human sciences and the philosophical currents such as dialectics and phenomenology have on a very narrow group of researchers that set up the field of Nutrition.

Key words: Qualitative research. Postgraduate programs. Master's. PhD. Nutrition. Social sciences.

\section{Introdução}

A emergência da Nutrição no Brasil como campo científico, política pública e profissão, fenômeno histórico relativamente recente, ocorreu a partir dos primeiros anos de 1930, tendo sido objeto de investigação de alguns estudos realizados nas últimas três décadas. ${ }^{1-7}$

Por sua vez, a emergência da pós-graduação em Nutrição no Brasil, fenômeno histórico muito mais recente, ocorreu a partir da criação do Curso de Mestrado em Nutrição da Universidade Federal de Pernambuco (UFPE), o qual teve seu início no primeiro semestre de $1971 .{ }^{8}$ De acordo 
com Kac et al., ${ }^{9}$ os programas de pós-graduação stricto sensu que compunham a subárea de Nutrição na Coordenação de Aperfeiçoamento de Pessoal de Nível Superior (Capes) passaram por crescimento expressivo na última década: correspondiam a cinco em 1995, chegando a 18 em 2011. Segundo dados da Capes, em 1ํo de julho de 2013, 23 programas e cursos de pós-graduação stricto sensu encontravam-se vinculados à área de avaliação de Nutrição (http://www.capes.gov. br/cursos-recomendados-e-reconhecidos).

Embora escassos, alguns estudos realizados na última década têm procurado analisar o ensino, a produção científica e outras características da pós-graduação no Brasil. Nunes et al., ${ }^{10}$ ao analisarem a trajetória da pós-graduação em Saúde Coletiva no Brasil, particularmente no período de 1996-2006, observaram que houve incremento do número de programas e cursos que se iniciaram na década de 1970, diversificação das denominações e áreas de concentração e continuidade do desequilíbrio regional, pois 66\% dos programas localizavam-se na região Sudeste.

Viacava, ${ }^{11}$ por sua vez, ao analisar a produção científica dos cursos de pós-graduação em Saúde Coletiva no período 1998-2006, tendo por base os artigos completos registrados no sistema Coleta da Capes, constatou expressivo crescimento da produção de artigos publicados em periódicos, os quais respondiam por cerca de $60 \%$ da produção científica da área, bem como observou considerável aumento da publicação de artigos em periódicos internacionais e nacionais com maior Qualis e, também, uma redução da publicação em revistas nacionais e locais sem Qualis.

Rosa \& Leta ${ }^{12}$ realizaram investigação procurando identificar qual a principal tendência epistemológica da pesquisa originada em 11 programas de pós-graduação em Educação Física no Brasil, baseando-se nos relatórios anuais de avaliação do triênio 2001-2003 da Capes. A análise da produção bibliográfica do triênio revelou maior prevalência de estudos com abordagem biológica, apoiados principalmente na Fisiologia. Entretanto, também identificou crescimento de estudos com fundamentação nas Ciências Sociais e Humanas, revelando a heterogeneidade epistemológica e a natureza multidisciplinar desse campo de conhecimento.

Araújo \& Almeida ${ }^{13}$ realizaram investigação das dissertações e teses produzidas no período de 1974 a 2004 nos programas de pós-graduação em Nutrição no Brasil, procurando traçar um perfil descritivo da produção científica sobre aleitamento materno. A análise dessa produção científica revelou uma tendência em estudar o aleitamento materno abordando apenas suas dimensões biológicas e epidemiológicas, sem se preocupar com as dimensões subjetivas, sociais e culturais que envolvem a sua prática.

Embora seu foco principal não seja a análise de dissertações e teses, destaca-se o estudo realizado por Canesqui, ${ }^{14}$ o qual constitui uma análise do estado da arte da pesquisa qualitativa em Alimentação e Nutrição publicada no período de 1985-2007 na base de dados Scientific Electronic Library Online (SciELO). De acordo com os resultados obtidos, dos 327 resumos capturados, 93 $(28,4 \%)$ se designavam como pesquisas qualitativas ou incorporaram referenciais das ciências 
sociais e humanas. A autora concluiu que as pesquisas qualitativas ampliaram positivamente a interlocução da Nutrição com as ciências sociais e humanas, embora requeiram aperfeiçoamento teórico-metodológico para superar os estudos descritivos, adequando o seu entendimento.

Publicado na década de 1980, o estudo de Rocha \& Silva ${ }^{15}$ destaca-se entre os demais pela tentativa de identificar as vinculações de 285 dissertações e teses produzidas nos programas de pós-graduação em Enfermagem às correntes de pensamento filosófico que as embasaram. À época, o estudo revelou uma influência marcante do positivismo e uma tendência emergente de outras correntes filosóficas, como, por exemplo, a dialética e a fenomenologia, nos trabalhos científicos de Enfermagem produzidos no Brasil.

O objetivo deste artigo é identificar e analisar as correntes filosóficas e metodológicas do conhecimento científico que embasaram as dissertações e teses com abordagens qualitativas e mistas produzidas nos programas de pós-graduação em Nutrição no Brasil no período de 2003 a 2012.

\section{Método}

Para definição dos programas de pós-graduação em Nutrição a serem investigados foram pré-estabelecidos os seguintes critérios de seleção: 1) Do universo de 22 programas e cursos de pós-graduação vinculados à área de Nutrição da Capes, em 15 de maio de 2013, deveriam ser incluídos apenas os programas compostos simultaneamente por cursos de mestrado acadêmico e doutorado (n=9) (http://www.capes.gov.br/cursos-recomendados); 2) Os programas a serem incluídos deveriam dispor de páginas eletrônicas que disponibilizassem a relação das dissertações e teses defendidas no período a ser investigado; 3) O período de investigação corresponderia aos últimos dez anos (janeiro de 2003 a dezembro de 2012).

Foram excluídos três dos nove programas pré-selecionados por não preencherem os requisitos de disponibilização da relação das dissertações e teses defendidas no período a ser investigado - os programas da Universidade de Brasília (UnB), da Universidade Estadual de Campinas (Unicamp) e da Universidade de São Paulo (USP). Foi intencionalmente incluído na investigação o Programa de Pós-Graduação em Alimentos, Nutrição e Saúde da Universidade Federal de Bahia (UFBA), em função de sua produção no campo da pesquisa qualitativa. Ao final da aplicação dos procedimentos de seleção foram definidos sete programas de pós-graduação em Nutrição a serem investigados: o da Universidade Federal de Pernambuco (UFPE), da Universidade Federal do Rio de Janeiro (UFRJ), da Universidade Federal de São Paulo (Unifesp), da Universidade Federal de Viçosa (UFV), da Universidade Federal de Santa Catarina (UFSC), da Universidade Federal da Bahia (UFBA) e da Universidade do Estado do Rio de Janeiro (Uerj). 
$\mathrm{O}$ acesso à relação das dissertações e teses foi feito a partir de visitas, entre os dias 15 e 31 de maio de 2013, às homepages dos sete programas de pós-graduação investigados. Cabe ressaltar que apenas quatro programas (Uerj, UFBA, UFRJ e UFSC) disponibilizavam em suas páginas eletrônicas, além das listagens por ano de defesa, links (atalhos) ou outros recursos para acesso aos resumos e/ou documentos na íntegra. Os três demais programas (UFPE, UFV e Unifesp) só disponibilizavam as listagens por ano de defesa, sendo assim, o acesso aos resumos e/ou documentos na íntegra, quando necessário, foi feito a partir de consulta ao Banco de Teses da Capes (http:// capesdw.capes.gov.br/capesdw/).

Para sistematizar o processo de categorização e análise, a relação das dissertações e teses de cada um dos sete programas investigados foi importada para planilhas do Microsoft Excel $^{\circledR}$, nas quais as informações disponíveis, tais como número de ordem, autor do trabalho, título, data de defesa, nome do orientador, banca examinadora, nível, linha de pesquisa, área de concentração e palavras-chave (informações variáveis para cada programa) foram reorganizadas de acordo com os interesses específicos da presente investigação.

Para a realização da análise, cada dissertação ou tese foi submetida a duas modalidades de categorização: 1) Quanto ao tipo de abordagem do conhecimento científico (ou correntes de pensamento filosófico-metodológico); e 2) Quanto aos núcleos de saber/conhecimento do campo da Nutrição.

Quanto às abordagens do conhecimento científico, as dissertações e teses foram categorizadas em três modalidades, a saber: abordagens quantitativas, abordagens qualitativas e abordagens quantiqualitativas ou mistas. Essa categorização foi baseada em estudos de autores vinculados à disciplina Metodologia Científica ou Metodologia da Pesquisa, tais como John W. Creswell, ${ }^{16}$ Marina de Andrade Marconi e Eva Maria Lakatos ${ }^{17}$ e Roberto Jarry Richardson. ${ }^{18}$ De fato, a intenção inicial era categorizar as dissertações e teses quanto às concepções ou correntes filosóficas que as embasavam, entretanto, frente à constatação de que para a grande maioria dos estudos tais concepções encontravam-se ocultas ou não explícitas, optamos por nos guiar na categorização proposta por Creswell. ${ }^{16}$ Assim, grosso modo, para Creswell, ${ }^{16}$ a abordagem quantitativa tem por finalidade testar teorias objetivas (dedutivas), examinar a relação entre variáveis que podem ser medidas por instrumentos e analisar os dados numéricos obtidos a partir de procedimentos estatísticos; a abordagem qualitativa tem por finalidade a exploração ou análise em profundidade, de forma indutiva, dos significados que o indivíduo ou grupos de indivíduos atribuem a um problema social ou humano e a abordagem mista, por sua vez, tem por finalidade envolver o uso simultâneo ou em conjunto das abordagens quantitativa e qualitativa, procurando potencializar a força geral ou o poder explicativo do estudo em relação ao uso isolado destas duas abordagens. 
Quanto aos núcleos de saber/conhecimento do campo da Nutrição, as dissertações e teses foram categorizadas em seis modalidades, a saber: a) Nutrição Clínica; b) Nutrição Básica e Experimental; c) Ciência e Tecnologia de Alimentos Aplicadas à Saúde; d) Nutrição em Saúde Coletiva; e) Ciências Sociais e Humanas em Alimentação e Nutrição; e f) Nutrição em Produção de Refeições. Essa categorização foi construída tendo por base os requisitos da área de avaliação de Nutrição da Capes para a organização das linhas e atividades de pesquisas nos programas de pós-graduação (http:// www.capes.gov.br/images/stories/download/avaliacao/Criterios_APCNs_Nutricao.pdf)e a compilação, realizada pelo Fórum Nacional de Coordenadores de Programas de Pós-Graduação em Alimentação e Nutrição, dos núcleos de saberes presentes nos programas de pós-graduação do campo Alimentação e Nutrição inseridos na área de avaliação de Medicina II na Capes, em 2009. ${ }^{9}$ Sendo assim, no núcleo de conhecimento Nutrição Clínica foram incluídas as dissertações e teses voltadas para o estudo de diagnósticos e/ou terapêuticas nutricionais das patologias humanas no âmbito individual. No núcleo de conhecimento Nutrição Básica e Experimental foram incluídas as dissertações e teses envolvendo pesquisas básicas de caráter experimental e laboratorial com animais e/ou seres humanos abrangendo os campos de Bioquímica, Dietética, Fisiologia, Genética, Patologia, entre outros. No núcleo de conhecimento Ciência e Tecnologia de Alimentos Aplicadas à Saúde foram incluídas dissertações e teses envolvendo pesquisas sobre composição química, qualidade sanitária, Engenharia e Tecnologia de Alimentos. No núcleo de conhecimento Nutrição em Saúde Coletiva foram incluídas dissertações e teses sobre diagnósticos e intervenções nutricionais, no âmbito coletivo, envolvendo disciplinas e conteúdos como Epidemiologia Nutricional, Políticas, Planejamento e Gestão em Alimentação e Nutrição, entre outras. No núcleo de conhecimento Ciências Sociais e Humanas em Alimentação e Nutrição foram incluídas dissertações e teses com abordagem nos campos da Antropologia, Comunicação, Direito, Economia, Educação, Filosofia, História, Geografia e Sociologia em Alimentação e Nutrição, entre outras. No núcleo de conhecimento Nutrição em Produção de Refeições foram incluídas as dissertações e teses sobre produção e consumo de refeições, sobre avaliação e proposição de sistemas de qualidade (nutricional, sensorial, higiênico sanitária, regulamentar, simbólica e sustentabilidade) em unidades de alimentação e nutrição coletivas e comerciais.

$\mathrm{Na}$ análise para categorização das dissertações e teses quanto às abordagens do conhecimento científico e aos distintos núcleos de saber/conhecimento do campo científico da Nutrição foram realizados os seguintes procedimentos: 1) Leitura dos títulos; 2) Identificação da(s) linha(s) de pesquisa(s) do orientador; 3) Leitura dos resumos; e 4) Leitura da seção/capítulo sobre os procedimentos metodológicos da investigação. Ressalta-se que para a grande maioria das dissertações e teses $(72,8 \%)$ apenas a leitura dos títulos foi suficiente para a realização dos procedimentos de categorização, uma vez que os títulos ofereciam informações adequadas para tal. 
Para análise das dissertações e teses categorizadas dentro de abordagens qualitativas e quantiqualitativas $(n=70)$ procedeu-se, além da leitura dos resumos e capítulos metodológicos, a identificação do referencial bibliográfico utilizado. Para sistematizar o processo de análise destas 70 dissertações e teses, procurou-se identificar: a) O tipo de abordagem ou desenho (explícito ou oculto) utilizado; b) Os procedimentos e instrumentos do processo de coleta de dados; c) Os procedimentos de análise dos dados; e d) Os referenciais teóricos principais e secundários utilizados na fundamentação científica do estudo. Ressalta-se que neste artigo a análise das abordagens qualitativas e mistas foi realizada de forma completa apenas para 43 dissertações e teses que se encontravam disponíveis na íntegra nas homepages dos programas da UFBA, Uerj e UFSC. As 20 dissertações e teses com abordagens qualitativas e mistas encontradas nos programas da UFPE, UFRJ, Unifesp e UFV não estavam disponíveis na íntegra e, portanto, não foi possível incluí-las nesta análise.

\section{Resultados}

Conforme tabela 1, para o conjunto de 962 dissertações e teses defendidas no período de 2003 a 2012 nos sete programas de pós-graduação investigados, observou-se a hegemonia da abordagem quantitativa, uma vez que este tipo de pesquisa foi adotado por $92,5 \%(n=890)$ do total de pesquisadores. Em termos numéricos, a abordagem qualitativa teve uma participação "marginal", uma vez que foi adotada por apenas 5,4\% $(n=52)$ do total de pesquisadores. As abordagens mistas ou quantiqualitativas, por sua vez, foram adotadas por apenas $1,9 \%(n=18)$ do total de pesquisadores.

Analisando-se os sete programas de forma isolada, observou-se que o perfil de hegemonia da pesquisa quantitativa se reproduziu para cada um deles, sendo quase exclusivo para os programas da Unifesp (99\%, n=103), UFPE (97,4\%, n=289), UFV (96,7\%, n=145) e UFRJ (95,1\%, n=155). A participação das abordagens qualitativas e mistas ocorreu de forma mais expressiva nos programas da UFBA ( $n=20,24,7 \%)$, Uerj $(n=13,28,3 \%)$ e UFSC $(n=17,14 \%)$. Nos quatro demais programas, a participação das abordagens qualitativas e mistas ocorreu de forma numérica inexpressiva, perfazendo 3,6\% $(n=6)$ na UFRJ, 3,3\% $(n=5)$ na UFV, 2,6\% $(n=8)$ na UFPE e $1 \%(n=1)$ na Unifesp (tabela 1). 
Tabela 1. Distribuição das dissertações e teses defendidas no período de 2003 a 2012 nos sete programas de pós-graduação em Nutrição investigados, segundo o tipo de abordagem do conhecimento científico. Florianópolis, SC, 2012.

\begin{tabular}{lccccc}
\hline PROGRAMA DE & \multicolumn{5}{c}{ Tipo de abordagem do conhecimento científico } \\
\cline { 2 - 6 } $\begin{array}{l}\text { PÓS-GRADUAÇÃO } \\
\text { EMUTRIÇÃO }\end{array}$ & $\begin{array}{c}\text { Quantitativa } \\
\mathrm{n}(\%)\end{array}$ & $\begin{array}{c}\text { Qualitativa } \\
\mathrm{n}(\%)\end{array}$ & $\begin{array}{c}\text { Quantiqualitativa } \\
\mathrm{n}(\%)\end{array}$ & $\begin{array}{c}\text { Outras } \\
\mathrm{n}(\%)\end{array}$ & $\begin{array}{c}\text { Total } \\
\mathrm{n}(\%)\end{array}$ \\
\hline UFPE & $289(97,4)$ & $4(1,3)$ & $4(1,3)$ & -- & $297(100,0)$ \\
UFRJ & $155(95,1)$ & $3(1,8)$ & $3(1,8)$ & $2(1,3)^{(*)}$ & $163(100,0)$ \\
Unifesp & $103(99,0)$ & $1(1,0)$ & -- & $104(100,0)$ \\
UFV & $145(96,7)$ & -- & $5(3,3)$ & -- & $150(100,0)$ \\
UFSC & $104(86,0)$ & $14(11,6)$ & $3(2,4)$ & -- & $121(100,0)$ \\
UFBA & $61(75,3)$ & $18(22,2)$ & $2(2,5)$ & -- & $81(100,0)$ \\
Uerj & $33(71,7)$ & $12(26,1)$ & $1(2,2)$ & -- & $46(100,0)$ \\
\hline TOTAL & $890(92,5)$ & $52(5,4)$ & $18(1,9)$ & $2(0,2)$ & $962(100,0)$ \\
\hline
\end{tabular}

(*) Trata-se de elaboração de material didático sobre educação alimentar e nutricional, o qual não se enquadrou nas categorizações de abordagem do conhecimento científico pré-estabelecidas.

Etnografias, hermenêutica e fenomenologia: características principais das abordagens qualitativas no Programa de Pós-Graduação em Alimentos, Nutrição e Saúde da UFBA

No programa de pós-graduação da UFBA, 14 dissertações se autocategorizaram como etnografias ou exercícios/estudos etnográficos. Os procedimentos de coleta de dados destas dissertações compreenderam: observação livre, observação participante, diários ou registros de campos, registros fotográficos, entrevistas semiestruturadas com gravação e, em uma delas, a realização de grupos focais. Portanto, tais procedimentos são coerentes e característicos das pesquisas com abordagem qualitativa. ${ }^{16-20}$

A chamada antropologia hermenêutica, ou simbólica ou interpretativa do antropólogo norteamericano Clifford James Geertz (1926-2006) (http://pt.wikipedia.org/wiki/Clifford_Geertz), 
constituiu o principal referencial de análise da maioria destas dissertações, sendo este autor referenciado em pelo menos 12 dissertações, a partir da obra $A$ interpretação das culturas. ${ }^{21}$

A chamada hermenêutica filosófica de Hans-Georg Gadamer (1900-2002) (http://pt.wikipedia. org/wiki/Hans-Georg_Gadamer), filósofo alemão autor de Verdade e método, ${ }^{22}$ apareceu como referencial interpretativo de pelo menos quatro dissertações.

A abordagem sobre pesquisa qualitativa da socióloga brasileira Maria Cecília de Souza Minayo, descrita principalmente em sua obra $O$ desafio do conhecimento: pesquisa qualitativa em saúde, ${ }^{19}$ constituiu referencial transversal de análise de pelo menos 16 dissertações.

A teoria das representações sociais do psicólogo social romeno Serge Moscovici apareceu como principal referencial interpretativo de duas dissertações, a partir da obra A representação social da psicanálise. ${ }^{23}$

A perspectiva hermenêutica e fenomenológica do filósofo francês Paul Ricœur (1913-2005) (http://pt.wikipedia.org/wiki/Paul_Ric\%C5\%93ur) apareceu como principal referencial de análise de uma dissertação, a partir da leitura de Do texto à ação: ensaios de hermenêutica II, ${ }^{24}$ embora duas outras dissertações também façam referência a esse autor.

O método da análise de discurso do filósofo francês vinculado à corrente do materialismo histórico Michel Pêcheux (1938-1983) (http://pt.wikipedia.org/wiki/Michel_P\%C3\%AAcheux) apareceu como estratégia analítica de uma dissertação. A análise semiótica do filósofo francês Roland Barthes (1915-1980) (http://pt.wikipedia.org/wiki/Roland_Barthes) apareceu como referencial analítico principal de uma dissertação e como referencial coadjuvante de pelo menos outras cinco, a partir da leitura de Elementos de semiologia. ${ }^{25}$

O método de história de vida do sociólogo francês Daniel Bertaux (http://en.wikipedia.org/ wiki/Daniel_Bertaux) apareceu como referencial analítico de duas dissertações, a partir da leitura de Narrativas de vida: a pesquisa e seus métodos. ${ }^{26}$

Esse conjunto de dissertações (etnografias) pode ser caracterizado como análises multirreferenciais, nas quais, grosso modo, se mesclam distintas perspectivas epistemológicas ou correntes filosóficas metodológicas do conhecimento científico. Assim, além dos referenciais analíticos já identificados, outros importantes autores transitaram nas análises dessas dissertações com abordagem qualitativa, alguns vinculados à abordagem fenomenológica, tais como Alfred Schutz (1899-1959) e Maurice Merleau-Ponty (1908-1961), outros vinculados à etnopsicanálise, como o antropólogo/filósofo francês François Laplantine, e à psicologia social, como Martin Bauer, outros vinculados ao estruturalismo, como o filósofo Michel Foucault (1926-1984) e ao estruturalismo construtivista, como Pierre Bourdieu (1930-2002).

Transitaram ainda como referenciais coadjuvantes das dissertações com abordagem qualitativa vários autores contemporâneos do emergente campo das Ciências Sociais e Humanas em 
Alimentação e Nutrição (Antropologia, Economia, Educação, Filosofia, Geografia, História, Sociologia, Psicologia, entre outras), ${ }^{14}$ entre estes destacam-se Claude Fischler, Jean Louis Flandrin, Jean Pierre Poulain, Jesús Contreras Hernández, Mabel Gracia Arnáiz e Massimo Montanari. Destacam-se ainda autores nacionais como Ana Maria Canesqui, Klaas Axel Anton Wessel Woortmann, Maria Eunice de Souza Maciel, Rosa Wanda Diez Garcia, Ligia Amparo da Silva Santos e Maria do Carmo Soares de Freitas, sendo as duas últimas, docentes permanentes do programa de pós-graduação da UFBA e responsáveis pela linha de investigação qualitativa baseada em etnografias.

Outra linha de investigação com abordagem qualitativa ou quantiqualitativa identificada nas dissertações defendidas no programa da UFBA tomou como objeto de investigação a construção e/ou aplicação de modelos teóricos de avaliação de políticas públicas. Esta linha foi identificada em pelo menos quatro destas dissertaçóes analisadas. Embora categorizadas dentro da pesquisa qualitativa e apresentando uma abordagem multirreferencial, em geral, estas dissertações não apresentavam, de forma explícita, um referencial teórico específico. Entre os referenciais teóricos identificados, destacamos o chamado liberalismo igualitário do filósofo político John Rawls (19212002) (http://pt.wikipedia.org/wiki/John_Rawls), a partir da leitura da obra Uma teoria da justiça ${ }^{27}$ e a chamada teoria da avaliação aplicada aos serviços e programas sociais baseada nos princípios democráticos e participativos e na utilidade social dos resultados do pedagogo social espanhol Miquel Gómez Serra (http://www.agapea.com/libros/Evaluacion-de-los-servicios sociales9788497840033-i.htm), a partir da leitura da obra Evaluación de los servicios sociales. ${ }^{28}$

Grupos focais, etnografias, estruturalismo construtivista: características principais das abordagens qualitativas no Programa de Pós-Graduação em Alimentação, Nutrição e Saúde da Uerj

No Programa de Pós-Graduação em Alimentação, Nutrição e Saúde da Uerj, das 13 dissertações analisadas, duas se autocategorizaram como etnografias, uma como estudo socioantropológico, uma como análise textual baseada na semiótica, uma como análise dos grupos de pesquisa no campo da Alimentação e Nutrição, uma como construção e aplicação de modelo teórico para avaliação de política pública e as demais, embora usando procedimentos de coleta e análise associados à pesquisa qualitativa, ${ }^{16-20}$ não explicitavam o tipo de desenho/estudo a que se afiliavam. Na homepage do programa, das 13 dissertações analisadas, apenas para cinco estavam disponibilizadas as versões completas dos trabalhos, para as demais estavam disponíveis apenas os resumos, os quais, em sua maioria, não apresentavam as informações necessárias para nossa análise. Os procedimentos de coleta de dados das duas etnografias foram descritos como observação sistemática, registros fotográficos e entrevistas etnográficas. Os procedimentos de coleta de dados das demais dissertações foram: duas utilizaram a combinação de entrevistas semiestruturadas com grupos focais; duas, a 
combinação de entrevistas semiestruturadas com observação participante; duas utilizaram apenas a realização de entrevistas semiestruturadas; duas, apenas a realização de grupos focais; uma utilizou os conteúdos textuais de capas de revista; uma utilizou as informações do Diretório dos Grupos de Pesquisa do CNPq; e uma utilizou uma combinação de procedimentos de coleta de dados (revisão de literatura, consulta a especialistas, etc.) para a construção do modelo avaliativo. Portanto, tais procedimentos são coerentes e característicos das pesquisas com abordagem qualitativa. ${ }^{16-20}$

A análise do referencial teórico-metodológico só foi possível ser realizada para nove das dissertações, as quais estavam disponibilizadas na íntegra $(n=5)$ ou os resumos ofereciam as informações mínimas necessárias $(n=4)$. Semelhante à análise realizada para as dissertações do programa da UFBA, observa-se que o conjunto dessas nove dissertações do programa da Uerj também pode ser caracterizado como análises multirreferenciais, nas quais se mesclam distintas perspectivas epistemológicas ou correntes filosóficas metodológicas do conhecimento científico.

O estruturalismo construtivista do filósofo/sociólogo francês Pierre Bourdieu (1930-2002) apareceu como principal referencial teórico de quatro dissertações e como referencial coadjuvante de mais duas, particularmente a adoção dos conceitos de habitus, campo científico e capital simbólico, feita a partir da leitura de obras como A economia das trocas simbólicas ${ }^{29}$ e Sobre a televisão. ${ }^{30}$

A abordagem sobre pesquisa qualitativa da socióloga brasileira Maria Cecília de Souza Minayo, presente principalmente em sua obra $O$ desafio do conhecimento: pesquisa qualitativa em saúde,$^{19}$ constituiu referencial transversal de análise de quatro dissertações.

O materialismo histórico-dialético apareceu como referencial teórico coadjuvante ou transversal em pelo menos duas dissertações, a partir de referência a autores vinculados a esta corrente do pensamento científico-filosófico, tais como o filósofo alemão Karl Marx (1818 -1883), o filósofo alemão Friedrich Engels (1820-1895), o filósofo húngaro Georg Lukács (1885-1971), o filósofo de origem tcheca Karel Kosík (1926-2003), o sociólogo jamaicano Stuart Hall e o filósofo soviético Alexandre Cheptulin.

A concepção sobre consumismo pós-moderno do sociólogo polonês Zygmunt Bauman (http:// pt.wikipedia.org/wiki/Zygmunt_Bauman) apareceu como referencial coadjuvante de duas dissertações, a partir da leitura de Vida para consumo: a transformação das pessoas em mercadorias. ${ }^{31}$

A teoria das representações sociais de Serge Moscovici23 ${ }^{23}$ associada ao método de análise de conteúdo de Laurence Bardin, ${ }^{32}$ apareceu como principal referencial interpretativo de uma dissertação, feita a partir da leitura do artigo da socióloga francesa Claudine Herzlich, "A problemática da representação social e sua utilidade no campo da doença"."33

Uma das etnografias analisadas traz com principal referencial metodológico as cinco fases de atividades alimentares propostas pelo antropólogo britânico Jack Goody, a partir da leitura de sua obra Cook, cuisine and class: a study in comparative sociology, ${ }^{34}$ ao mesmo tempo em que também 
se fundamenta em clássicos da Antropologia e das Ciências Sociais e Humanas como o sociólogo e antropólogo francês Marcel Mauss (1872-1950), o antropólogo polonês Bronislaw Malinowski (1884-1942), o antropólogo franco-belga Claude Lévi-Strauss (1908-2009), o antropólogo norteamericano Clifford James Geertz (1926-2006) e outros, como os contemporâneos Tim May, ${ }^{35}$ Paul Atkinson e Martyn Hammersley. ${ }^{36}$

De modo semelhante ao verificado na análise do programa da UFBA, transitaram ainda como referenciais coadjuvantes das dissertações com abordagem qualitativa defendidas na Uerj vários autores contemporâneos do emergente campo das Ciências Sociais e Humanas em Alimentação e Nutrição, ${ }^{14}$ tais como Claude Fischler, Jean Pierre Poulain, Jesús Contreras Hernández, Mabel Gracia Arnáiz e Massimo Montanari.

Destacam-se ainda autores nacionais como Ana Maria Canesqui, José Ricardo de Carvalho Mesquita Ayres, Klaas Axel Anton Wessel Woortmann, Luciene Burlandy, Maria Eunice de Souza Maciel, Maria Lúcia Magalhães Bosi, Rosa Wanda Diez Garcia, Rosana Magalhães, Maria Cláudia da Veiga Soares Carvalho, Shirley Donizete Prado e Silvia Angela Gugelmin, sendo as três últimas, docentes vinculadas ao programa de pós-graduação da Uerj e responsáveis pela linha de investigação qualitativa.

Estudos de caso, Sociologia da Alimentação e Nutrição em produção de refeições: características principais das abordagens qualitativas no Programa de Pós-Graduação em Nutrição da UFSC

No Programa de Pós-Graduação em Nutrição da UFSC, das 17 dissertações analisadas, 15 se autocategorizaram como estudos de caso, uma como etnografia e uma como estudo fenomenológico. Na homepage do programa, para três dissertações não estavam disponibilizadas as versões completas dos trabalhos e na leitura dos resumos disponíveis no Banco de Teses da Capes não foram encontradas todas as informações necessárias para nossa análise. Os procedimentos de coleta de dados das dissertações categorizadas como estudos de caso compreenderam: análise documental, entrevistas semiestruturadas com gravação e observação direta $(n=6)$; análise documental, entrevistas semiestruturadas, observação direta e grupos focais $(\mathrm{n}=2)$; análise documental, observação direta, observação armada (medição, gravação, filmagem, exame clínico/ físico, degustação) e outras técnicas de coleta de dados $(\mathrm{n}=3)$; análise documental, elaboração, aplicação e avaliação de método ou procedimento técnico $(n=4)$. Os procedimentos de coleta de dados da etnografia centraram-se na observação participante e do estudo fenomenológico na realização de entrevistas com gravação. Portanto, tais procedimentos são coerentes e característicos das pesquisas com abordagem qualitativa. ${ }^{16-20}$ 
A análise do referencial teórico-metodológico só foi possível ser realizada para 14 das dissertações, as quais estavam disponibilizadas na íntegra. Semelhante à análise realizada para as dissertações dos programas da UFBA e da Uerj, observa-se que o conjunto dessas dissertações do programa da UFSC também pode ser caracterizado como análises multirreferenciais, nas quais se mesclam distintas perspectivas epistemológicas ou correntes filosóficas metodológicas do conhecimento científico. Ressalta-se que todas as dissertações categorizadas como estudos de caso têm vinculação com o núcleo de conhecimento Nutrição em Produção de Refeições, uma linha de pesquisa de destaque do Programa de Pós-Graduação da UFSC. Sendo assim, em geral, tais dissertações tiveram como bases empíricas de investigação unidades de produção de refeições (UPR) de hospitais, indústrias, restaurantes, hotéis, escolas, etc. Em pelo menos nove desses estudos de caso, as obras Inovação tecnológica na produção de alimentação coletiva ${ }^{37}$ e $O$ espaço social alimentar: um instrumento para o estudo dos modelos alimentares ${ }^{38}$ constituíram o referencial teórico-metodológico que caracteriza a especificidade do núcleo de conhecimento Nutrição em Produção de Refeições.

O principal referencial teórico-metodológico destes estudos de casos foi a obra Manual de investigação em Ciências Sociais dos cientistas sociais belgas Raymond Quivy e Luc Van Campenhoudt, ${ }^{39}$ citado em pelo menos dez dissertações.

A pedagoga e doutora em Educação Arilda Schmidt Godoy apareceu como referencial em pelo menos nove dissertações. ${ }^{40,41}$

A abordagem sobre pesquisa qualitativa da socióloga brasileira Maria Cecília de Souza Minayo, principalmente de sua obra $O$ desafio do conhecimento: pesquisa qualitativa em saúde,$^{19}$ constituiu referencial transversal de análise de pelo menos seis dissertações.

A obra Estudo de caso: planejamento e métodos, de Robert K. Yin, ${ }^{42}$ apareceu como referencial de quatro dissertações.

O método de análise de conteúdo de Laurence Bardin, ${ }^{32}$ apareceu como principal referencial interpretativo de quatro dissertações.

Ressalta-se que, além destes referenciais teórico-metodológicos já identificados, outros importantes autores estrangeiros e nacionais transitaram nas análises deste conjunto de dissertações do programa de pós-graduação da UFSC como forma de fundamentação científica da pesquisa qualitativa, entre os quais se destacam: Antônio Carlos Gil (Brasil, Ciências Sociais, Pedagogia), Antônio Chizzotti (Brasil, Educação), Augusto Nibaldo Silva Triviños (Chile/Brasil, Pedagogia e Filosofia), Flick Uwe (Berlim/Canadá, Psicologia, Sociologia), George Gaskell (Inglaterra, Psicologia Social), José Carlos Zanelli (Brasil, Psicologia, Educação), Martin W. Bauer (Inglaterra, Psicologia Social), Peter Henry Mann (1926-2008, Inglaterra, Sociologia) e Roberto Jarry Richardson (Chile/ Brasil, Sociologia). 
O estudo com abordagem fenomenológica fez referência aos autores clássicos da fenomenologia, tais como Edmund Husserl (1859-1938), Martin Heidegger (1889-1976) e Maurice Merleau-Ponty (1908-1961), entretanto, usou como base de fundamentação teórica obras de autores nacionais vinculados a esta corrente filosófica, tais como Adriano Furtado Holanda, ${ }^{43}$ Daniel Augusto Moreira,${ }^{44}$ Mauro Martins Amatuzzi ${ }^{45}$ e Yolanda Cintrão Forghieri. ${ }^{46}$

Por sua vez, o estudo etnográfico referiu como principal referencial analítico a chamada antropologia hermenêutica, ou simbólica ou interpretativa do antropólogo norte-americano Clifford James Geertz (1926-2006), além de referenciais coadjuvantes, tais como obras de antropólogos brasileiros como Roberto Cardoso de Oliveira (1928-2006) ${ }^{47}$ e Roberto Augusto Damatta. $^{48}$

De forma semelhante à verificada na análise dos programas da UFBA e Uerj, transitaram ainda como referenciais coadjuvantes das dissertações com abordagem qualitativa defendidas na UFSC vários autores contemporâneos do emergente campo das Ciências Sociais e Humanas em Alimentação e Nutrição, tais como Claude Fischler, Jean Pierre Poulain, Jean Louis Flandrin, Jesús Contreras Hernández, Mabel Gracia Arnáiz e Massimo Montanari.

Destacam-se ainda autores nacionais como Ana Maria Canesqui, Ligia Amparo da Silva Santos, Maria Eunice de Souza Maciel, Maria do Carmo Soares de Freitas, Maria Lúcia Magalhães Bosi, Rosa Wanda Diez Garcia, Shirley Donizete Prado, Silvia Angela Gugelmin, Anete Araújo de Sousa, Francisco de Assis Guedes de Vasconcelos e Maurício Soares Leite, sendo os três últimos, docentes do programa de pós-graduação da UFSC, vinculados à linha de investigação qualitativa.

\section{Considerações finais}

À guisa de conclusão, cabe explicitar os referenciais teóricos ou as concepções que embasaram este estudo. Sob a orientação teórica da sociologia reflexiva ou do estruturalismo construtivista de Pierre Bourdieu (1930-2002), tomamos emprestados os conceitos de campo científico, capital científico, capital simbólico, homo academicus e habitus científico. ${ }^{29,49,50}$ Simultaneamente, também nos guiamos pelos conceitos de paradigma, revolução científica e comunidade científica oriundos da filosofia idealista de Thomas Samuel Kuhn (1922-1996). ${ }^{51,52}$

A hegemonia da abordagem quantitativa, adotada por $92,5 \%(n=890)$ do total de dissertações e teses analisadas, denota por si a forte influência que as correntes filosóficas baseadas no positivismo e nas Ciências Biológicas ${ }^{16-20}$ continuam exercendo na conformação do campo científico da Nutrição. A influência desse paradigma positivista e biológico não é específica do campo da Nutrição, tendo sido identificada nos demais campos científicos relacionados às Ciências da Vida, tais como em Medicina, ${ }^{53}$ Odontologia, ${ }^{54}$ Enfermagem ${ }^{15}$ e Educação Física,,${ }^{12}$ entre outros. 
A participação "marginal" e "emergente" das abordagens qualitativas e mistas, adotada por apenas 7,3\% $(\mathrm{n}=70)$ do total de dissertações e teses analisadas, expressa por si a influência que as Ciências Sociais e Humanas e as correntes filosóficas, como a dialética e a fenomenologia, têm exercido sobre um grupo muito restrito de pesquisadores e cientistas que conformam o campo científico da Nutrição. Ressalta-se, novamente, que essa participação contra-hegemônica das abordagens qualitativas centradas em novos e alternativos paradigmas que fazem interfaces com as Ciências Sociais e Humanas e as correntes filosóficas, como a dialética e a fenomenologia, também tem sido identificada nos demais campos científicos relacionados às Ciências da Vida, tais como em Medicina, ${ }^{53}$ Odontologia, ${ }^{54}$ Enfermagem ${ }^{15}$ e Educação Física, ${ }^{12}$ entre outros.

A produção ou a reprodução do conhecimento científico em Nutrição centrada na adoção de um paradigma dominante, no caso positivista e biológico, por quase a totalidade dos praticantes dessa ciência, suscita o debate em torno das concepções de comunidade científica e campo científico, conforme propostas por Kuhn ${ }^{51,52}$ e Bourdieu, ${ }^{29,49,50}$ respectivamente. Neste momento, optamos por nos afastar da noção de que a comunidade científica seria uma estrutura autônoma e autorreprodutora, em que os cientistas cooperariam de forma neutra e desinteressada em nome do progresso científico. ${ }^{51,52}$ Preferimos nos aproximar da abordagem de campo científico como um mercado particular dentro da ordem econômica capitalista, caracterizado pela luta concorrencial travada entre cientistas e/ou instituições científicas em torno da posse do capital científico - uma espécie de capital simbólico, não monetário, e que se transforma em autoridade ou competência científica para os praticantes da ciência. ${ }^{29,49,50}$ Sendo assim, é preciso identificar nas atuais condições objetivas e subjetivas que determinam a conformação do campo científico da Alimentação e Nutrição no Brasil, quais os elementos que estão motivando o "interesse" da grande maioria dos praticantes dessa ciência em se afiliar ao paradigma dominante. Dentro de uma complexa rede de determinação, poderíamos apontar: a) Os estímulos financeiros oriundos das agências de fomento públicas e privadas, as quais, quase sempre, estabelecem como prioritários projetos de pesquisas focados no paradigma positivista e biológico; b) As características do sistema de avaliação do ensino e da pesquisa na pós-graduação, o qual é centrado em critérios quantitativos, índices de produtividade, citação, visibilidade e circulação do conhecimento produzido; e c) A luta concorrencial entre os cientistas do campo no processo de produção e distribuição do capital científico, a qual é sempre definida pelo estado de relação de forças entre os protagonistas das disputas, envolvendo elementos, como autoridade, hierarquia, dogmatismo, legitimidade, reconhecimento social e acúmulo do capital simbólico. ${ }^{29,49,50}$ Tais questões extrapolam os limites do presente estudo, demandando a realização de investigações futuras. 
A análise das 43 dissertações e teses com abordagens qualitativas e mistas produzidas nos três programas de pós-graduação investigados permite a seguinte síntese conclusiva:

1. Com relação ao desenho ou tipo de estudo, observou-se uma diferenciação entre os três programas investigados, sendo a modalidade etnografia hegemônica na UFBA, o estudo de caso hegemônico na UFSC e a metodologia de grupos focais prevalente na Uerj;

2. Com relação aos procedimentos de coleta e análise de dados, observou-se uma convergência entre os três programas na utilização de estratégias e procedimentos metodológicos coerentes e característicos das pesquisas com abordagem qualitativa; ${ }^{16-20}$

3. Com relação às principais correntes filosóficas que embasaram as dissertações e teses, observouse que, grosso modo, os três programas convergem na adoção de análises multirreferenciais, denotando a heterogeneidade epistemológica do campo da pesquisa qualitativa em Nutrição. Características específicas foram observadas para os três programas investigados: na UFBA predominou a adoção de referenciais vinculados às correntes filosóficas hermenêutica e fenomenologia; na Uerj, o estruturalismo construtivista foi a corrente filosófica mais prevalente; e na UFSC, a Sociologia da Alimentação constituiu o referencial teórico-metodológico que caracterizou a especificidade da pesquisa no campo da Nutrição em Produção de Refeições;

4. Ressalvadas as especificidades de cada programa, observou-se que os três comungam um leque bem expressivo de referenciais teóricos estrangeiros e nacionais vinculados tanto às distintas correntes filosóficas do conhecimento científico quanto ao emergente campo das Ciências Sociais e Humanas em Alimentação e Nutrição.

Na tentativa de melhor analisar esse conjunto de dissertações e teses com abordagens qualitativas e mistas produzidas nos três programas de pós-graduação analisados, voltamos a nos aproximar de alguns elementos da concepção de comunidade científica proposta por Kuhn. ${ }^{51,52}$ De acordo com esse autor, para compreender o conhecimento científico como propriedade intrínseca de uma determinada comunidade científica, torna-se necessário conhecer as características essenciais dessas unidades que o criam e o utilizam. Sendo assim, em linhas gerais, a organização e funcionamento de uma comunidade científica ocorreria da seguinte forma:

Uma comunidade científica é formada pelos praticantes de uma especialidade científica. Estes foram submetidos a uma iniciação profissional e a uma educação similares, numa extensão sem paralelos na maioria das outras disciplinas. Neste processo absorveram a mesma literatura técnica e dela retiraram muitas das mesmas lições. Normalmente as fronteiras dessa literatura-padrão marcam os limites de um objeto de estudo científico e em geral cada comunidade possui um objeto de estudo próprio (Kuhn,,$^{51}$ p. 220). 
Portanto, esses pesquisadores vinculados às abordagens qualitativas e mistas constituiriam comunidade(s) científica(s) por compartilhar o(s) mesmo(s) paradigma(s) (conjunto de crenças, valores, teorias, técnicas, procedimentos metodológicos, etc., capazes de fornecer problemas e soluções modelares para os praticantes da ciência). ${ }^{51,52}$

Por outro lado, as características comuns (procedimentos de coleta e análise de dados, tendência às análises multirreferenciais, socialização de referenciais teóricos estrangeiros e nacionais) e as especificidades (predominância de determinadas correntes filosóficas e metodológicas) identificadas nos três programas investigados também nos aproxima do conceito de habitus científico proposto por Bourdieu. ${ }^{29,49,50}$ Habitus científico compreendido como um sistema flexível, relacional, processual, inacabado e em constante transformação, constituído por disposições individuais construídas em condições históricas e socais específicas de existência, dentro de uma relação dialética entre indivíduo (interioridade/subjetividade) e sociedade (exterioridade/objetividade), sendo direcionado para funções e ações do agir científico. ${ }^{29,49,50}$

Em síntese, o campo da pesquisa qualitativa nos programas de pós-graduação em Nutrição apresenta características comuns que contribuem para a construção de um padrão identitário dos pesquisadores vinculados a este campo específico dentro do campo mais amplo da Alimentação e Nutrição brasileira. Simultaneamente, observam-se características distintas que podem contribuir para relações de concorrência/dependência/interdependência entre habitus institucionais e habitus científicos dos agentes sociais que diametralmente ocupam posições no campo. Caracteriza-se pela utilização de modelos analíticos multirreferenciais, podendo seu posicionamento "marginal" em relação ao status da pesquisa quantitativa ser explicado em função de múltiplos fatores, entre os quais se destacam a disponibilidade de recursos financeiros e materiais, a visibilidade da produção científica e o acúmulo do capital científico dos agentes sociais envolvidos.

\section{Referências}

1. L’Abbate S. As Políticas de Alimentação e Nutrição no Brasil: I. Período de 1940 a 1964. Rev Nut. 1988;1(2):87-138.

2. Ypiranga L. Delimitação do objeto de trabalho do nutricionista: subsídios para uma discussão. Saúde em Debate. 1990;(29):62-9.

3. Lima ES. Gênese e constituição da educação alimentar: a instauração da norma. Hist Ciên SaúdeManguinhos. 1998;5(1):57-84.

4. Vasconcelos FAG. Combate à fome no Brasil: uma análise histórica de Vargas à Lula. Rev Nut 2005;18(4):439-57. 
5. Vasconcelos FAG. Fome, eugenia e constituição do campo da nutrição em saúde pública em Pernambuco: uma análise de Gilberto Freyre, Josué de Castro e Nelson Chaves. Hist Ciên SaúdeManguinhos 2001. 8(2):315-39.

6. Vasconcelos FAG, Calado CLA. Profissão nutricionista: 70 anos de história no Brasil. Rev Nut. 2011;24(4):605-17.

7. Vasconcelos FAG, Batista Filho M. História do campo da Alimentação e Nutrição em Saúde Coletiva no Brasil. Ciên Saúde Coletiva 2011;16(1):81-90.

8. Vasconcelos FAG. Um perfil de Nelson Chaves e da sua contribuição à nutrição em saúde pública no Brasil. Cad Saúde Pública 2001;17(6):1505-18.

9. Kac G, Proença RPC, Prado SD. A criação da área “nutrição” na Capes. Rev Nut. 2011;24(6):905-16.

10. Nunes ED, Ferreto LE, Barros NF. A pós-graduação em Saúde Coletiva no Brasil: trajetória. Ciên Saúde Coletiva 2010;15(4):1923-34.

11. Viacava F. Produção científica dos cursos de pós-graduação em Saúde Coletiva no período 1998-2006. Ciên Saúde Coletiva 2010;15(4):1977-88.

12. Rosa S, Leta J. Tendências atuais da pesquisa brasileira em Educação Física: parte 2: a heterogeneidade epistemológica nos programas de pósgraduação. Rev Bras Educ Fís Esp. 2011;25(1):7-18.

13. Araújo RMA, Almeida JAG. O aleitamento materno na pós-graduação em nutrição no Brasil: um perfil das dissertações e teses de 1974 a 2004. Rev Bras Saúde Matern Infant. 2008;8(1):125-33.

14. Canesqui AM. Pesquisas qualitativas em nutrição e alimentação. Rev Nut. 2009;22(1):125-39.

15. Rocha SMM, Silva GB. Linhas filosóficas e ideológicas na pesquisa em enfermagem no Brasil. Rev Bras Enferm. 1987;40(4):214-21.

16. Creswell JW. Projeto de pesquisa: métodos qualitativo, quantitativo e misto. 3. ed. Porto Alegre: Artmed; 2010.

17. Marconi MA, Lakatos EV. Metodologia Científica. 6. ed. São Paulo: Atlas; 2011.

18. Richardson RJ. Pesquisa Social: Métodos e Técnicas. 3. ed. São Paulo: Atlas; 1999.

19. Minayo MCS. O desafio do conhecimento: pesquisa qualitativa em saúde. 9. ed. São Paulo: HUCITEC; 2006.

20. Minayo MCS. Pesquisa Social: teoria, método e criatividade. 27. ed. Rio de Janeiro: Vozes; 2008.

21. Geertz C. A Interpretação das Culturas. Rio de Janeiro: LTC; 1989.

22. Gadamer HG. Verdade e Método. Traços fundamentais de uma hermenêutica filosófica. Petrópolis: Vozes; 1997.

23. Moscovici S. A representação social da psicanálise. Rio de Janeiro: Zahar; 1978.

24. Ricoeur P. Do texto a acção. Ensaios de hermenêutica II. Porto: Rés; 1989.

25. Barthes R. Elementos de Semiologia. São Paulo: Cultrix; 1997. 
26. Bertaux D. Narrativas de vida: a pesquisa e seus métodos. Natal: EDUFRN; São Paulo: Paulus; 2010.

27. Rawls J. Uma teoria da justiça. Lisboa: Editorial Presença; 1993.

28. Serra MG. Evaluación de los servicios sociales. Barcelona: Editorial Gedisa; 2004.

29. Bourdieu P. A economia das trocas simbólicas. 6. ed. São Paulo: Perspectiva; 2005.

30. Bourdieu P. Sobre a televisão. Rio de Janeiro: Zahar; 1997.

31. Bauman Z. Vida para consumo: a transformação das pessoas em mercadorias. Rio de Janeiro: Zahar; 2008.

32. Bardin L. Análise de conteúdo. 3. ed. Lisboa: Edições 70; 2009.

33. Herzlich C. A problemática da Representação Social e sua utilidade no campo da doença. Physis (Rio J.) 2005;15(supl.):57-70.

34. Goody J. Cook, cuisine and class. A study in comparative sociology. 6. ed. New York: Cambridge University Press; 1996.

35. May T. Pesquisa social: questões, métodos e processos. 3. ed. Porto Alegre: Artmed; 2004.

36. Atkinson P, Hammersley M. Ethnography and Participant Observation. In: Denzin NK, Lincoln YS (orgs.). Handbook of Qualitative Research. London: Sage Publications; 2000. p. 248-61.

37. Proença RPC. Inovação tecnológica na produção de alimentação coletiva. Florianópolis: Insular; 1997.

38. Poulain JP, Proença RPC. O espaço social alimentar: um instrumento para o estudo dos modelos alimentares. Rev Nut. 2003;16(3):245-56.

39. Quivy R, Campenhoudt LV. Manual de investigação em Ciências Sociais. Lisboa: Gradiva; 1992.

40. Godoy AS. Introdução à pesquisa qualitativa e suas possibilidades. Rev Adm Empres. 1995;35(2):57-63.

41. Godoy AS. Pesquisa qualitativa: tipos fundamentais. Rev Adm Empres. 1995;35(3):20-9.

42. Yin RK. Estudo de caso: planejamento e métodos. 3. ed. Porto Alegre: Bookman; 2005.

43. Bruns MAT, Holanda AF. Psicologia e Fenomenologia: reflexões e perspectivas. Campinas: Alínea; 2003.

44. Moreira DA. O método fenomenológico na pesquisa. São Paulo: Pioneira Thomson; 2002.

45. Amatuzzi MM. O resgate da fala autêntica: filosofia da psicoterapia e da educação. Campinas: Papirus; 1989.

46. Forghieri YC. Psicologia fenomenológica: fundamentos, método e pesquisa. 2. ed. São Paulo: Pioneira; 2004.

47. Cardoso de Oliveira R. O trabalho do antropólogo. São Paulo: UNESP: Paralelo; 1998.

48. Da Matta R. Relativizando: uma introdução à antropologia social. Petrópolis: Vozes; 1987.

49. Bourdieu P. O poder simbólico. Rio de Janeiro: Bertrand Brasil; 1998.

50. Bourdieu P. Para uma Sociologia da Ciência. Lisboa: Biblioteca 70; 2008. 
51. Kuhn TS. A estrutura das revoluções científicas. 5. ed. São Paulo: Perspectiva; 1997.

52. Kuhn TS. O caminho desde A Estrutura. São Paulo: UNESP; 2006.

53. Tesser CD, Luz MT. Uma introdução às contribuições da epistemologia contemporânea para a Medicina. Ciênc Saúde Coletiva. 2002;7(2):363-72.

54. Gomes D, Da Ros, MA. A etiologia da cárie no estilo de pensamento da ciência odontológica. Ciên Saúde Coletiva. 2008;13(3):1081-90.

Recebido: $13 / 7 / 2013$

Aprovado: 15/8/2013 